\title{
Robotic Vehicle Control using Internet via Webpage and Keyboard
}

\author{
Ketan Dumbre \\ Department of Electronics \\ and Telecommunication \\ RSCOE, Pune-33
}

\author{
Snehal Ganeshkar \\ Department of Electronics \\ and Telecommunication \\ RSCOE, Pune-33
}

\author{
Ajinkya Dhekne \\ Department of Electronics \\ and Telecommunication \\ RSCOE, Pune-33
}

\begin{abstract}
Security is the need of the day. Terrorist attacks are on a rise throughout the world. This has led to an increasing need for surveillance, which is a very daunting task. There are surveillance cameras in some areas, but they have a very limited vision. This is not of much use as the view can get obstructed easily, which has provided an impetus to build a robotic vehicle for surveillance purposes. This robotic vehicle will be controlled via internet thus increasing the area it surveys. The keyboard manoeuvred surveillance vehicle will also have a robotic arm mounted on it. The robotic arm should essentially help pick and place or move objects whenever necessary.
\end{abstract}

\section{Keywords}

Wireless communication, Raspberry-pi, Webpage Control, Servo motor, Webcam.

\section{INTRODUCTION}

The rapid growth of industry and advancement of technology has resulted in reduction of human efforts, the main reason for which being machines!! Machines are playing an important role in our life. A machine might be anything, be it a cell phone or a bike or even a robot. [1][2] Robots have found an increasing demand in a wide range of applications in our life. Their use in defense has increased by the day. Our paper includes one such instance of how a bot can be of use to human race in general. Robots ensemble human beings in many ways be it looks or functioning, but previously robots were not controlled by computer programs or electronic circuitry. Back then they were built using principle of mechanics improving over time with the coming of electronic age.

In today's world, robots find use in various places be it to detonate buried bombs or in industrial applications or even robotic components used in children's toys. The complexity of computer software depends on how difficult the robot's tasks are.

In this project we use internet to establish communication between the user and a robotic vehicle. This is a reliable connection and a continuous video feedback is available to control the robotic vehicle. Due to the use of internet, there is no limitation on range or distance between the user and the robotic vehicle. Internet robotics has opened up a completely new range of real-world applications namely tele-surgery, tele-manufacturing, tele-training, telesurgery, traffic control, health care, space exploration, disaster rescue etc. and the list is supposed to increase further in the coming years.[3]

In previous projects, many researchers have developed a system for automatic control using ZigBee, GSM or Wi-Fi modules. [4][5] Almost all systems are wired, but now we have tried the same by the use of internet. The papers we have referred to convey the use of following techniques: In Wi-Fi Robot for Video Monitoring \& Surveillance System, the proposed robot motion will be controlled with PWM techniques using a Microcontroller and Bidirectional DC Bridge for Motor Driving. It is proposed to address the low cost, efficient, high speed processing \& control hardware for the self-navigating robotics application. [6]

Design and Implementation of a Robotic Vehicle with Real-Time Video Feedback Control via Internet paper illustrates on an approach to control a robotic vehicle using internet as communication medium between user and robotic vehicle. Conventionally wireless robots have the drawbacks of limited working range, limited frequency range and limited control. But internet can overcome these limitations with the help of DTMF decoder IC and a preprogrammed microcontroller IC that control the movement of the robotic vehicle according to output sent from DTMF decoder. [7].

\section{METHODOLOGY}

The webcam will capture live data with regards to its surroundings and then send it to a desired device through internet. The user will be observing this data on the monitor at the user end. According to the desired movement, the user will control the robotic vehicle and the robotic arm through the webpage or keyboard available at the user end. The input given through the webpage or the keyboard is then sent through the internet and the desired movement occurs at the robot end.

\subsection{Proposed Block Diagram}

The first part is construction of the robotic vehicle. With the help of programs an internet connection is established between the robotic vehicle and the user. Then robot captures the images using a webcam and stores them into the memory. The next task is to capture and send live images using internet at a rate sufficient to make them seem like a live video to the human eye. This was initially implemented using LAN before moving to internet. The desired result was achieved by sending compressed low resolution images so that transmission would not be affected in case high upload speeds were not available. Then the program was made more dynamic by varying the resolution of the images to be transmitted depending on the upload speed available at that particular time. Like for example in case of availability of good upload speeds, high resolution images will be sent and vice versa in case of low upload speeds. 


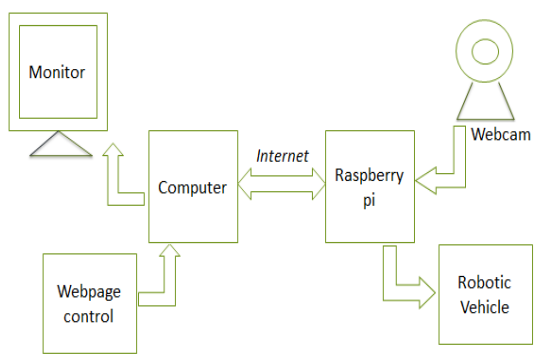

Figure 1.Block Diagram

\section{DESIGN AND IMPLEMENTATION 3.1 Raspberry Pi}

Raspberry $\mathrm{Pi}$ is used for making robot wireless and web based.[8] Webcam is interfaced to the Raspberry Pi and then the videos are transmitted wirelessly from the robot to the user's monitor, from where the user can conveniently control the robotic vehicle's movement and also the robotic arm movement. Raspberry pi is connected with the dongle which enables raspberry pi to transmit over the web network.

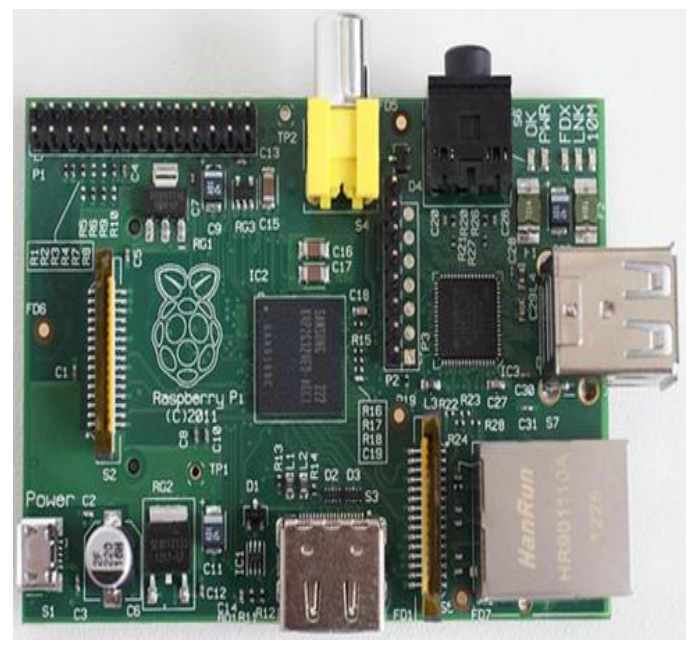

Figure 2. Raspberry-Pi Module

Raspberry Pi uses an SD card for booting and for memory as it doesn't have an inbuilt hard disk for storage. Raspberry Pi requires 5 volt supply with minimum of 700$1000 \mathrm{~mA}$ current and it is powered through micro USB cable. ARM11 only requires 3.3 volt of supply which it takes with the help of linear regulator. 5 volt is required for the USB ports. It operates at $700 \mathrm{MHz}$ We use python or embedded $\mathrm{C}$ to write the code into the raspberry pi. It has a strong processing capability due to the ARM11 architecture and Linux-based system. In terms of interface and control, it has 1 SPI, 1 UART, 1 I2C and 8 GPIO, which basically meet the control requirement. There are easy to use open source peripheral driver libraries. [9]

\subsection{Robotic Arm}

It should have three rotational joints along with a gripper. The gripper will open and close by means of the gear wheels. The base rotates in circular direction and the other two joints for upward, downward and forward, backward motion respectively. There is a limit to the movement each joint can produce since each joint is controlled by a servo motor. [10]

\subsection{Web Camera}

The visual feedback is provided by the Intex IT-306WC webcam. It can have a resolution of up to 30.0MP, Frame rate of 30FPS along with night time vision. It is plugged into the USB port of the Raspberry Pi.

\subsection{Motor Driver Circuit}

This circuit consists of the motor driver IC 1293d used to power the DC motors. [11] These DC motors will be used to maneuver the robotic vehicle.

\subsection{Servo Motor Pulse Width}

Conversion of angle in degrees to pulse width in $\mathrm{m} \mathrm{sec}$

Max pulse width (180 degree): $2.1 \mathrm{msec}$

Min pulse width (0 degree): $0.7 \mathrm{~m} \mathrm{sec}$

Angle entered: a

$\therefore$ pulse width $=[(2.1-0.7) *(\mathrm{a} / 180)]+0.7$

E.g. If $a=90$,

$\therefore$ pulse width $=[(2.1-0.7) *(90 / 180)]+0.7$

$$
\begin{aligned}
& =[1.4 * 0.5]+0.7 \\
& =1.4 \mathrm{msec}
\end{aligned}
$$

The result holds true for all angles and we can determine what pulse width to be given to a servo when a particular angle is entered.

\subsection{Software Design}

Python programming is used here. [12] Software design is divided into 4 codes namely:

\subsubsection{Webcam Server}

Webcam Server is the code run in the Raspberry Pi to capture the images and stream them over the internet. Here the images will be compressed into .jpg format to reduce their size prior to their transmission over the internet. They are sent using byte array over the UDP socket. [13]

\subsubsection{Webcam Client}

Webcam Client is the run in by the user to receive this images in the form of byte array. The are then displayed on the monitor at a rate closer to 12-20 images per second so that they appear like a continuous video.[14]

\subsubsection{Motor Server}

Motor Server is run by the user. Monitoring the video, the user maneuvers the robotic vehicle or the robotic arm accordingly. This is done by accepting input either from the keyboard or the webpage. It is done by checking the key press events.

\subsubsection{Motor Client}

As per the input from the user, either the robotic vehicle or the robotic arm move. This is done by making High or Low the desired GPIO pins of the Raspberry Pi. 4 GPIO pins are connected to the 4 servo motors and 4 to the motor driver IC 1293d.[15]

\subsection{Flowchart}

Flow of events is shown in diagram below: 

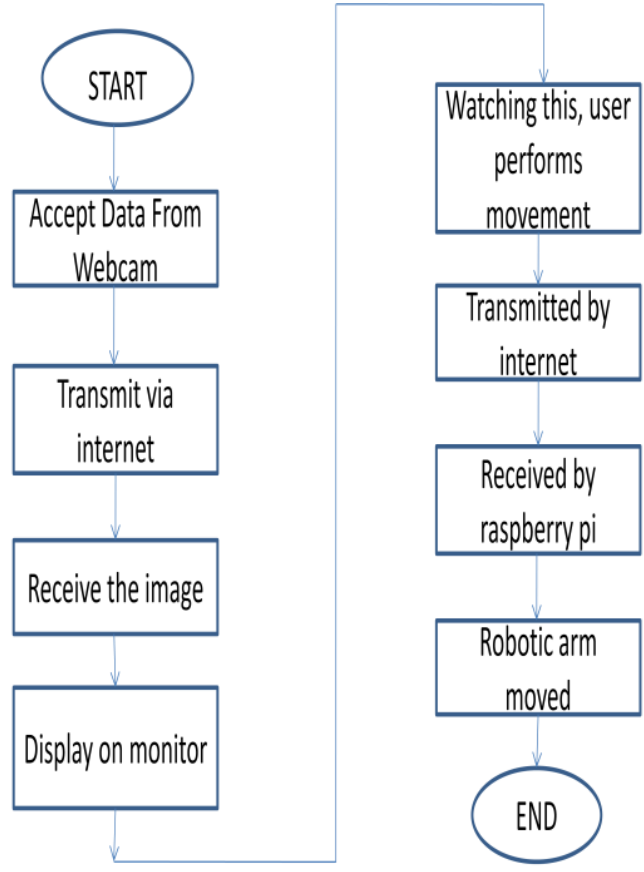

Figure 3. Flow chart

Flowchart is shown in Figure 3.It explains the manner in which actions will takes place here. So, initial stage is to capture the data with the help of a webcam. Then that data will be transmitted to the monitor side. According to presented situation, user will takes necessary actions like picking or placing any object or it can be movement of vehicle. But it will be in terms of signals, which will be transmitted through the internet. When these signals are received by the raspberry pi placed on vehicle, the robotic arm and vehicle will move accordingly. [16]

Again webcam will capture and send images to the user to take action. This cycle will go on.

\section{RESULTS}

Depending on the data rates available, the image quality and the resolution can be varied. When high data rates are available, we may send high quality and high resolution images as shown in Figure 6, but when high data rates not available we may have to send low quality and low resolution images.

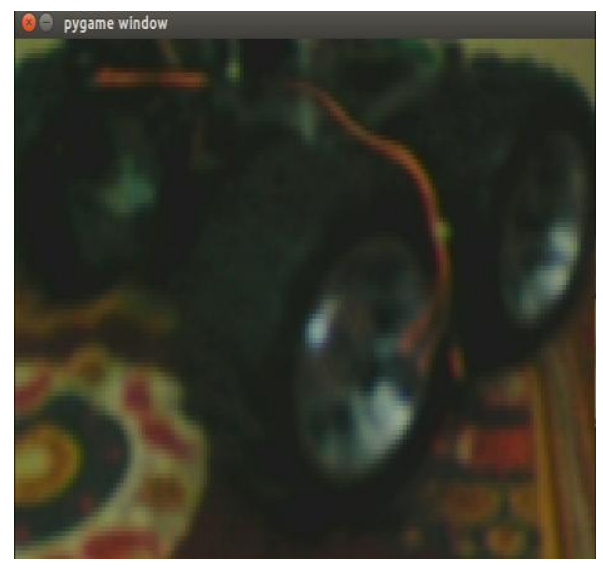

Figure 4. Image of resolution $160 * 120$

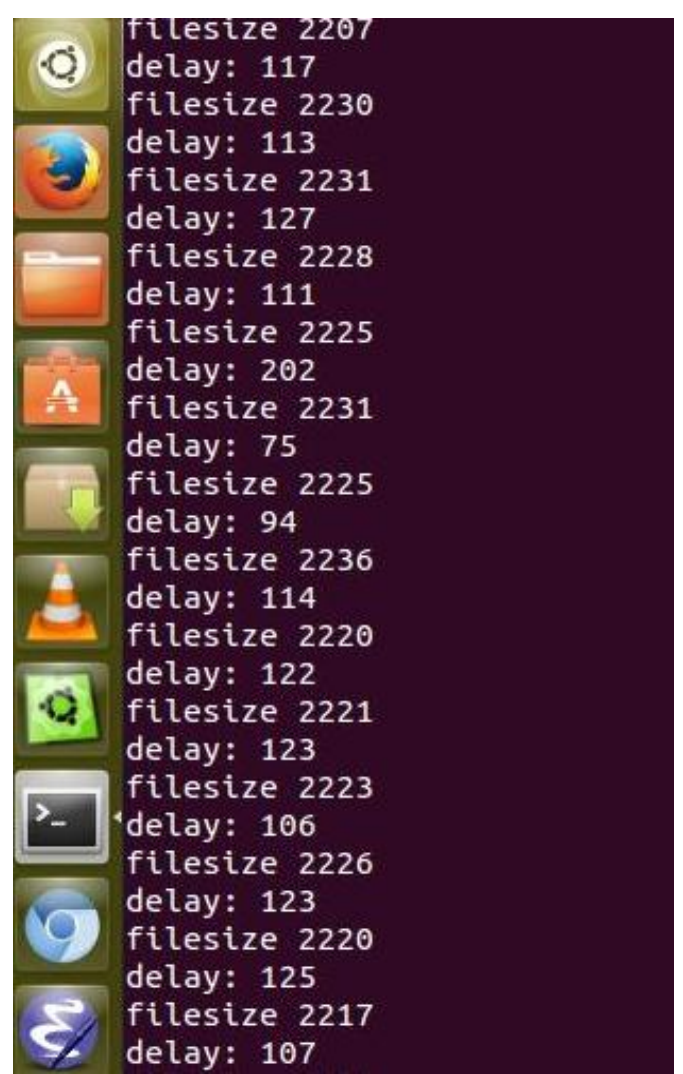

Figure 5. Delay for sending image of resolution $160 * 120$

Thus the user always has a visual feedback for maneuvering the surveillance vehicle (see Figure 4).

From Figure 5 and Figure 7, we can conclude that the delay is increasing with the increase in the file size. The increase in file size is a result of increased resolution. So to reduce this delay, there is a need for higher upload speeds.

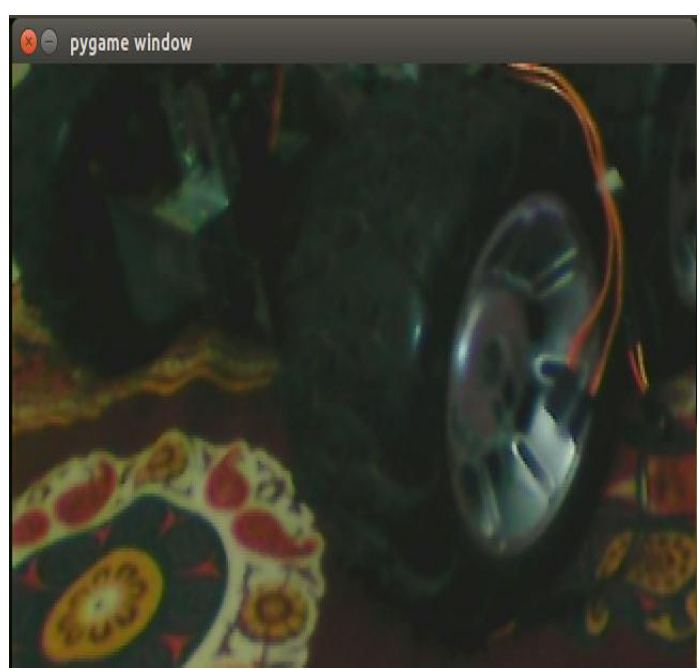

Figure 6. Image of resolution $320 * 240$ 


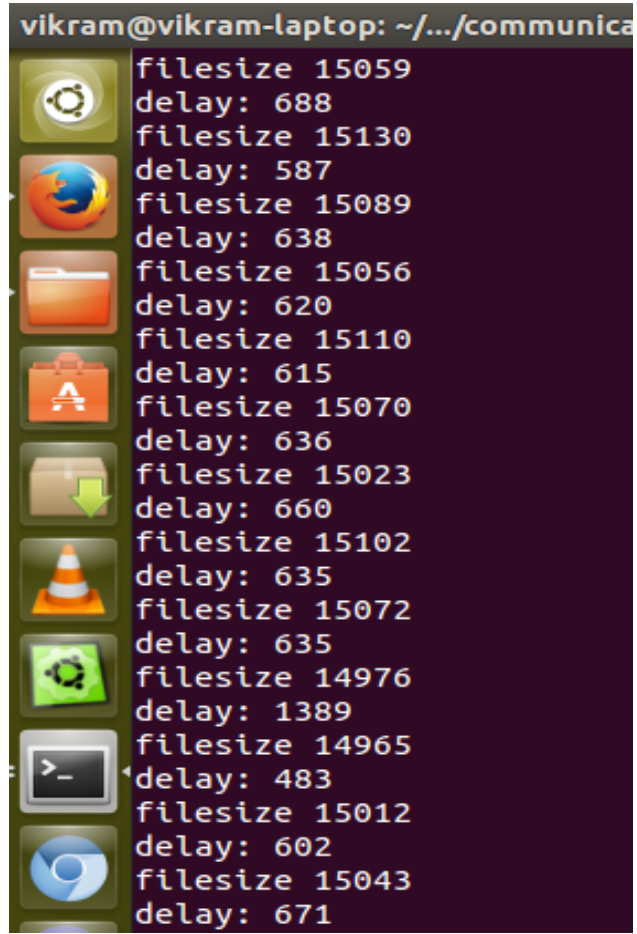

Figure 7. Delay for sending image of resolution $320 * 240$

Note: These readings are for upload speeds in the range of $50-100 \mathrm{kbps}$.Better quality images of much higher resolution can be sent with availability of higher upload peeds.

\begin{tabular}{|l|l|l|}
\hline Resolution & $\mathbf{1 2 0 * 1 6 0}$ & $\mathbf{3 2 0 * 2 4 0}$ \\
\hline Avg. Delay & 114 & 619.3 \\
\hline
\end{tabular}

Figure 8. Observation Table

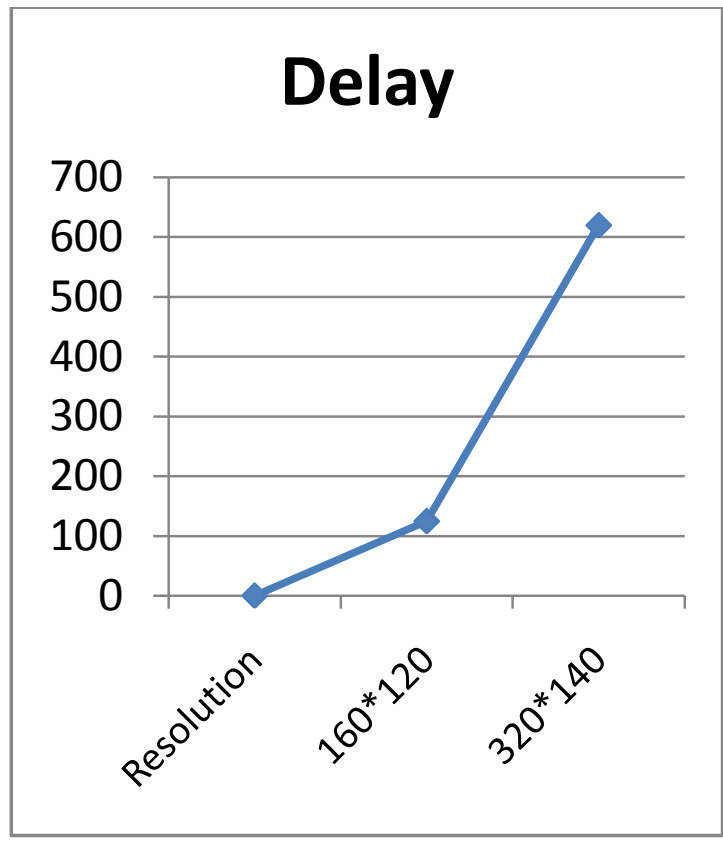

Figure 9. Resolution Vs Delay

\section{CONCLUSION}

Paper contains detailed information for controlling a robotic vehicle guided via internet. It has been done with the use of TCP and UDP protocols for transport layer data transfer. The size of the images sent by the robotic vehicle is controlled by a feedback. This feedback signal determines whether the size of the image should be small or large depending on the upload speed available with the Raspberry pi, thus making it much more dynamic to stream live visual data along with the successful movement of the robotic vehicle and the robotic arm.

Faster communication will ensure that we can send high quality, high resolution images with minimal delay or latency. This will help reduce delay in execution of commands providing real time access to the robot. It will be of great use for monitoring illegal activities occurring around us. It will also be useful in disaster affected areas to find and rescue injured people. It would also be used for spy bot.

\section{ACKNOWLEDGMENTS}

We thank Mr. B.D. Jadhav for his support and guidance throughout the study. We would also like to thank our mentor Mr. Vikram Bhat for guiding us throughout the project.

\section{REFERENCES}

[1] P. Saucy and F. Mondana," Keep On The Web: Open access to a mobile robot on the Internet", IEEE Robotics and Automation Magazine, pages 41-47, March 2000.

[2] R.C. Luo, T.M. Chen, and C.C. Yih, "Intelligent autonomous mobile robot control through the Internet,"IEEE International Symposium ISIE 2000, vol. 1, pp. 6-11, December 2000

[3] Huosheng Hu, Lixiang Yu, Pui Wo Tsui, Quan Zhou, "Internet-based Robotic Systems for Teleoperation", International Journal of Assembly Automation, Vol. 21, No. 2.

[4] Krishnaswamy Kannan and Gowtham S, “ Intelligent Personal Assistant and Surveillance Robot using Zigbee Communication",International Journal of Engineering Science and Technology (IJEST), ISSN : 0975-5462 Vol. 4 No.10 October 2012.

[5] G. Arangurenss, L. Nozal, A. Blazquez, and J. Arias, "Remote control of sensors and actuators by GSM", IEEE 2002 28th Annual Conference of the Industrial Electronics Society IECON 02,vol,5-8 Nov. 2002,pp.2306 - 2310

[6] Pavan C \& Dr. B. Sivakumar, "Wi-Fi Robot for Video Monitoring \& Surveillance System" International Journal of Scientific \& Engineering Research Volume 3, Issue 8, August-2012.

[7] G. Song, Z. Wei, W. Zhang and A. Song, "A hybrid sensor network system for home monitoring applications", IEEE Trans Consum Electron, Vol. 53, No. 4,pp. 14341439, 2007

[8] Priyanka .M and Raja ramanan .V, "Unmanned Aerial Vehicle for Video Surveillance Using Raspberry Pi", International Journal of Innovative Research in Science, Engineering and Technology Volume 3, Special Issue 3, March 2014 
[9] Dr. Shantanu K.Dixit and Mr. S. B. Dhayagonde, "Design and Implementation of e-Surveillance Robot for Video Monitoring and Living Body Detection", International Journal of Scientific and Research Publications, Volume 4, Issue 4, April 2014

[10] V. Ramya, B. Palaniappan, T. Akilan "Embedded System for Robotic Arm Movement Control using Web Server and ZigBee Communication", International Conference on Research Trends in Computer Technologies (ICRTCT - 2013)

[11] S.M. Tasmeeh Ahsan, Md. Zahid Hassan, Md. Tareq Imam,“ Design and Implementation of a Robotic Vehicle With Real-Time Video Feedback Control Via Internet", International Conference on Electrical Engineering and Information \& Communication Technology (ICEEICT) 2014

[12] D.M.Beazely,"Scientific Computing with Python", Astronomical Data Analysis Software and Systems IX, eds. N. Manset, 2000, in ASP Conf. Ser, Vol. 216
[13] Zhao Zhang "'The Internet Remote Robot with Skype Webcam",International Conference on System Science and Engineering,June 30-July 2, 2012, Dalian, China

[14] W. Lao, J. Han and Peter H.N. de With, "Automatic video-based humanmotion analyzer for consumer surveillance system", IEEE Trans Consum Electron, Vol. 55, No. 2, pp. 591-598, 2009.

[15] Aakanksha Pimpalgaonkar, Mansi Jha, Nikita Shukla „Kajol Asthana, "A Precision Temperature Controller Using Embedded System",International Journal of Scientific and Research Publications, Volume 3, Issue 12, December 2013

[16] V. Ramya, T. Akilan and B. Palaniappan,"Embedded System for Robotic Arm Movement Controlusing Web Server and ZigBee Communication", International Conference on Research Trends in Computer Technologies(ICRTCT-2013),Proceedings published in International Journal of Computer Applications $®(I J C A)(0975-8887) 30$ 